\title{
TU/e EmonOWEN

\section{On the optimal radiation bandwidth of printed slot antennas surrounded by EBGs}

\section{Citation for published version (APA):}

Neto, A., Llombart, N., Gerini, G., \& de Maagt, P. J. I. (2006). On the optimal radiation bandwidth of printed slot antennas surrounded by EBGs. IEEE Transactions on Antennas and Propagation, 54(4), 1074-1083. https://doi.org/10.1109/TAP.2006.872586

DOI:

10.1109/TAP.2006.872586

Document status and date:

Published: 01/01/2006

\section{Document Version:}

Publisher's PDF, also known as Version of Record (includes final page, issue and volume numbers)

\section{Please check the document version of this publication:}

- A submitted manuscript is the version of the article upon submission and before peer-review. There can be important differences between the submitted version and the official published version of record. People interested in the research are advised to contact the author for the final version of the publication, or visit the $\mathrm{DOI}$ to the publisher's website.

- The final author version and the galley proof are versions of the publication after peer review.

- The final published version features the final layout of the paper including the volume, issue and page numbers.

Link to publication

\section{General rights}

Copyright and moral rights for the publications made accessible in the public portal are retained by the authors and/or other copyright owners and it is a condition of accessing publications that users recognise and abide by the legal requirements associated with these rights.

- Users may download and print one copy of any publication from the public portal for the purpose of private study or research.

- You may not further distribute the material or use it for any profit-making activity or commercial gain

- You may freely distribute the URL identifying the publication in the public portal.

If the publication is distributed under the terms of Article 25fa of the Dutch Copyright Act, indicated by the "Taverne" license above, please follow below link for the End User Agreement:

www.tue.nl/taverne

Take down policy

If you believe that this document breaches copyright please contact us at:

openaccess@tue.nl

providing details and we will investigate your claim. 


\title{
On the Optimal Radiation Bandwidth of Printed Slot Antennas Surrounded by EBGs
}

\author{
Andrea Neto, Member, IEEE, Nuria Llombart, Student Member, IEEE, Giampiero Gerini, Member, IEEE, and \\ Peter De Maagt, Senior Member, IEEE
}

\begin{abstract}
This paper describes a design strategy to achieve the maximum bandwidth and efficiency for a printed slot antenna surrounded by EBGs. First the dielectric constant and the thickness of the dielectric slab that guarantees an acceptable front to back radiation ratio is identified. Then electromagnetic bandgap (EBG) structures are designed to achieve the optimal bandwidth (BW) while obtaining high surface wave efficiency in the radiating half space. To achieve this goal the wave interaction between the slot and the EBG structures is investigated in depth and clearly described. For the case of Planar circularly symmetric (PCS) EBGs the maximum of radiation $\mathrm{BW}$ is shown to occur when the distance between the central antenna and the EBG is approximately half wavelength of the first surface wave, $\lambda_{\mathrm{sw}} / 2$.
\end{abstract}

Index Terms-Electromagnetic bandgap (EBG), planar circularly symmetric electromagnetic bandgap (EBG), planar printed antennas, slot antennas, surface waves, wideband antennas.

\section{INTRODUCTION}

$\mathbf{T}$ HE design of antennas surrounded by electromagnetic band gap (EBG) structures has been the object of many investigations (see [1] for an overview). In many cases the introduction of the EBG materials was aimed at enhancing the directivity [2] of the antenna, at reducing the mutual coupling [3] or at increasing the scan performances in arrays [4]. When the antenna and EBG are printed on the same slab, a problem that systematically arises is choice of the optimal distance between the antenna and the surrounding EBG. In [5] simulations for different distances have been presented showing a significant impact of this parameter on the input impedance. In [6], the fact that the distance parameter should be selected to avoid reactive coupling between the antenna and the EBG was emphasized.

This paper describes a design strategy to achieve the maximum bandwidth and efficiency, in terms of both front to back

Manuscript received August 29, 2005; revised November 30, 2005. This work was developed under contract with the European Space Agency (ESTEC contract ESTEC 17 539/03/NL/JAK "Validated Electromagnetic Modeling of Metal-Dielectric Photonic Bandgap (PBG) Structures."

A. Neto is with the Defence, Security and Safety Institute, Netherlands Organization for Applied Scientific Research (TNO), Den Haag 2597 AK, The Netherlands (e-mail: andrea.neto@tno.nl).

N. Llombart is with the Departamento de Comunicaciones, Universidad Politécnica de Valencia, E-46022, Valencia, Spain on leave from the Defence, Security and Safety Institute, Netherlands Organization for Applied Scientific Research (TNO), Den Haag 2597 AK, The Netherlands.

G. Gerini is with Defence, Security and Safety Institute, Netherlands Organization for Applied Scientific Research (TNO), Den Haag 2597 AK, The Netherlands (e-mail: giampiero.gerini@tno.nl).

P. De Maagt is with the Electromagnetics Division, European Space Research and Technology Centre (ESTEC), 2200 AG, Noordwijk, The Netherlands (e-mail: Peter.de.Maagt@esa.int).

Digital Object Identifier 10.1109/TAP.2006.872586 ratio and surface wave suppression, for a printed slot antenna surrounded by EBGs. When a slot antenna is printed on a surface-wave less substrate, the real part of the admittance of the slot becomes the key parameter, since it is essentially associated only to power radiated in space. In this situation, the input impedance BW corresponds to the radiation BW.

In Section II, assuming that surface waves are not launched, the front to back radiation ratio of the slot is traded off with radiation BW using the dielectric constant and the thickness of the slab as design parameters. Unfortunately substrates that do not support surface waves do not exist and, even assuming an ideal EBG, this condition would only be fulfilled at one single frequency.

Also ideal EBG structures do not exist, and thus the remaining part of the paper is devoted to the design of a realistic EBG structure that minimizes, over a certain frequency band, the surface waves excited by a central slot antenna. In particular, in Sections III and IV, it is described how the distance between the antenna and the EBG can be selected to control the impact of the EBG on the antenna admittance. A simplified analytical model of the interaction between the antenna and the EBG is introduced. The simplification consists of considering a two dimensional problem that is infinitely extended in one dimension and that represents the interaction between the antenna and the EBG via a single surface wave mode.

The applicability of the single mode representation of the field is discussed in Section V. In that section a real slot antenna excited via microstrip and surrounded by a planar circularly symmetric (PCS) EBG, Fig. 1, has been simulated via full wave tools, for different values of the distance $\rho_{1}$. The wave interaction described in this paper provides a clear physical explanation for the increment in BW that was already anticipated in [7].

\section{SUbSTRATE SELECTION}

The slot, in absence of the EBG, is shown in Fig. 2. It is etched on a ground plane that divides two dielectric slabs with the same dielectric constant $\epsilon_{r}$ and different heights $h$ and $h_{m}$. The length and width of the slot are $l_{s}$ and $w_{s}$, respectively. The slot is excited via a microstrip whose extension beyond the slot crossing is $l_{\text {stub }}$ while its width is $w_{m}$.

The efficiency of printed antennas using elementary dipoles was already discussed in [8]. In this section, this discussion is extended to introduce a BW versus front to back ratio trade off in absence of surface waves. This condition will be then realized via EBGs. 


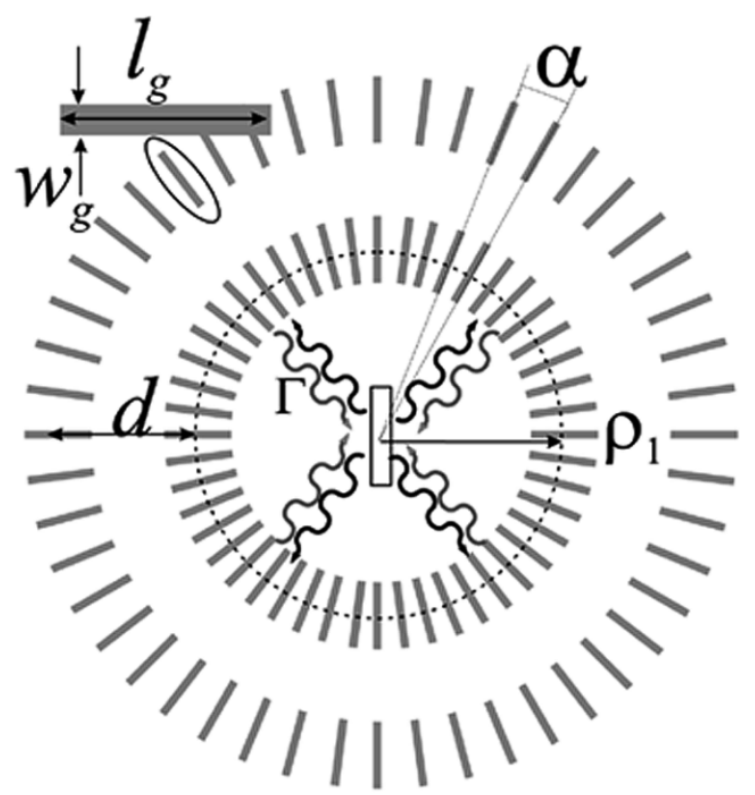

Fig. 1. Geometrical parameters of a PCS-EBG structure.

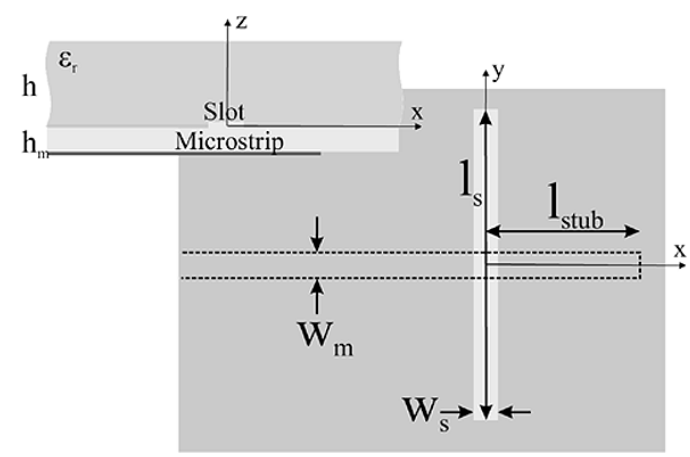

Fig. 2. Side and top view of the printed antenna: Slot $\left(l_{s}, w_{s}\right)$ coupled to a microstrip $\left(l_{\mathrm{stub}}, w_{m}\right)$.

The optimal substrate thickness and dielectric constant, from the point of view of radiation bandwidth, can been determined by just considering the properties of radiation of an elementary slot in the upper half space alone, characterized by $\epsilon_{r}, h$. The power radiated in the lower half space by an elementary magnetic dipole located on the ground plane can be approximated as the one radiated in a free half space. This is due to the fact that the thickness of the lower dielectric slab is taken in the order of $\lambda_{0} / 100$, where $\lambda_{0}$ is the free space wavelength. The power radiated in the upper half space, divided by the power radiated in the lower half space is plotted in Fig. 3, as a function of the thickness of the slab in terms of the wavelength in the dielectric $\left(\lambda_{d}\right)$, for $\epsilon_{r}=9.8$ (continuous line). This normalization of the power allows to directly obtain a measure of the front to back radiation of the slot. The graph also shows the power launched in the first surface waves, normalized to the downward radiated power. Similar graphs can be obtained for different values of the dielectric constant.

From Fig. 3 one can observe that the curve of the power radiated by a slot has a peak for thickness $h \approx\left(\left(\lambda_{d}\right) / 4\right)$. In this condition the front to back radiation ratio of the antenna is optimum but the efficiency of the antenna is very low since the

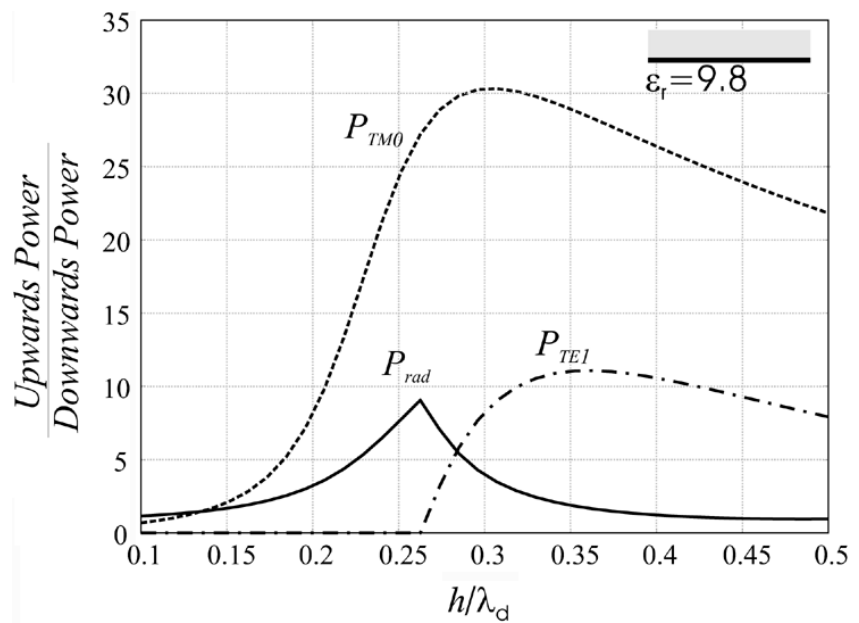

Fig. 3. Power radiated by an elementary magnetic dipole located on a ground plane underlying a slab of $\epsilon_{r}=9.8$. The power is plotted as a function of the height in terms of the wavelength in the dielectric $\left(\lambda_{d}\right)$ and divided by power that the slot would radiate in free space.

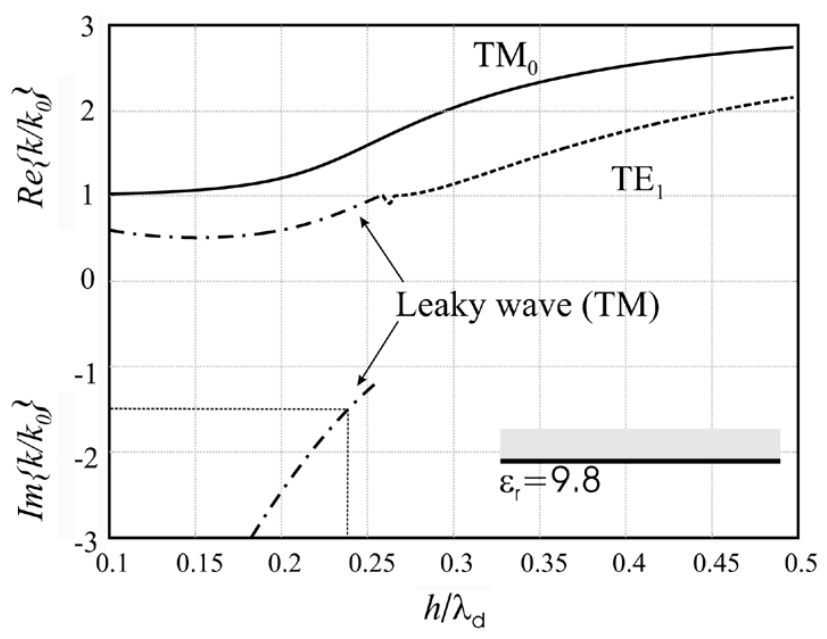

Fig. 4. Dispersion equation for a grounded slab of $\epsilon_{r}=9.8$ as a function of the thickness of the slab in terms of the wavelength in the dielectric.

surface waves are strongly excited. If one defines the surface wave efficiency of the slot $\eta_{S W}$ as

$$
\eta_{\mathrm{SW}}=\frac{P_{\mathrm{rad}}}{P_{\mathrm{rad}}+P_{\mathrm{SW}}}
$$

the efficiency of a slot, printed on a $\epsilon_{r}=9.8$ slab, has acceptable values (larger than 80\%) only when the dielectric slab is $h / \lambda_{d}<$ 0.1 , and consequently the front to back radiation ratio is small. For these reasons slot antenna designs are typically done for substrate parameters that provide a high surface wave efficiency but at the cost of a poor front to back ratio.

\section{A. Surface-Wave Less Substrate}

The front to back ratio of slot antennas could be greatly enhanced if one was able to suppress both TE and TM surface waves. Let us assume for the moment that this situation can be realized. The dispersion diagram in Fig. 4 shows that, besides the first surface waves, $\mathrm{TM}_{0}$ and $\mathrm{TE}_{1}$, also a $\mathrm{TM}$ leaky wave is present before the $\mathrm{TE}_{1}$ enters in propagation. The leaky pole seems to become less relevant for slabs thicker than $0.26 \lambda_{d}$, and 
our search algorithms could not track it any more after it merges with the branch in $k_{0}$. In a certain frequency range, typically just before the cutoff of the $\mathrm{TE}_{1}$ mode, the leaky wave radiation cannot be neglected and can give rise to significant pattern deformations. This pattern deformation is related to a low value of the attenuation constant of the leaky mode, $\left|\mathrm{IM}\left[k_{L W}\right]\right|<1.5 k_{0}$. Accordingly, both a deformation of the radiation pattern, and an increase of the mutual coupling between elements in array environment would be unavoidable if one operated the slot at a frequency at which the slab thickness is around $\lambda_{d} / 4$. In the example of Fig. 4, the thicknesses from $h / \lambda_{d}=0.239$ to $h / \lambda_{d}=$ 0.26 are associated with strong leaky wave effects.

Such a leaky wave is difficult to suppress due to the fact that its wave velocity is significantly different from the one of the $\mathrm{TM}_{0}$ surface wave. For this reason, slab thicknesses that would support leaky waves must be avoided. In the example of Fig. 4, the slab can be chosen with normalized thickness $h / \lambda_{d}$ either smaller than 0.239 or larger than 0.26 . The former case is convenient since it supports only the existence of the $\mathrm{TM}_{0}$ wave, while the latter would support both $\mathrm{TM}_{0}$ and $\mathrm{TE}_{1}$. Considering that suppressing only one mode is easier, the slab's thickness in this paper is selected to be $h / \lambda_{d}<0.239$.

This choice does not imply any sacrifice in terms of radiation $\mathrm{BW}$ in comparison with the thicker slab alternative, $h / \lambda_{d}>$ 0.26 . In fact, the maximum radiation $\mathrm{BW}$ that can be achieved is proportional only to the derivative of the radiated power curve in Fig. 3. This is approximately the same for the two alternative ranges of the dielectric slab thickness.

Let us now quantify the maximum radiation $\mathrm{BW}$ associated with the optimal substrate for a slot antenna in a surface-wave less substrate considering only the radiation contribution associated to the space wave emanating from the slot directly. For frequencies where the leaky wave is negligible, $\left|\mathrm{IM}\left[k_{L W}\right]\right|>1.5 k_{0}$, the space wave power is proportional to the radiated power curve of Fig. 3. Consequently, the space wave slot conductance associated to the upper media, normalized to the downward conductance, can be seen as the normalized power

$$
P_{\text {rad }}=G_{\text {rad }}
$$

One can then assume $-10 \mathrm{~dB}$ as acceptable reflection coefficient $\Gamma$ at the point of connection between the slot and a transmission line $(|\Gamma|<0.316)$.

The maximum height of the dielectric slab that allows to neglect the leaky wave radiation is $h=0.239 \lambda_{d}$ (see Fig. 4). One then obtains the corresponding maximum normalized conductance, $G_{\mathrm{rad}}^{\max }$, from Fig. 3. From this value and the requirement on the reflection coefficient amplitude, it is possible to derive the normalize characteristic admittance of the best matching transmission line

$$
G_{\text {line }}=G_{\text {rad }}^{\max } \frac{1-|\Gamma|}{1+|\Gamma|} .
$$

The minimal value of the normalized conductance, $G_{\mathrm{rad}}^{\mathrm{min}}$, that gives the same reflection coefficient can be expressed as

$$
G_{\mathrm{rad}}^{\min }=G_{\text {line }} \frac{1-|\Gamma|}{1+|\Gamma|} .
$$

TABLE I

MaXimum Conductances for Slots IN THE PRESENCE OF SuRface WaVe LESS Slabs FOR SEVERAL DieleCtric CONSTANTS

\begin{tabular}{|c|c|c|c|c|}
\hline$\epsilon_{r}$ & 4 & 9.8 & 16 & 25 \\
\hline$G_{\text {rad }}^{\max }$ & 2.1 & 6.46 & 11.9 & 23.8 \\
\hline
\end{tabular}

Accordingly, the relative radiation BW can be expressed in terms of the relative variation of the normalized slab's thickness

$$
2 \frac{f_{\max }-f_{\min }}{f_{\max }+f_{\min }}=2 \frac{h / \lambda_{d-\min }-h / \lambda_{d-\max }}{h / \lambda_{d-\min }+h / \lambda_{d-\max }}
$$

where $\lambda_{d-\min }$ corresponds to $f_{\max }$, the higher operational frequency defined by $G_{\mathrm{rad}}^{\max }$, and $\lambda_{d-\max }$ corresponds to the minimal operational frequency associated to $G_{\mathrm{rad}}^{\mathrm{min}}$.

Following this procedure, the maximum theoretical bandwidths for a surface-wave less substrate with $\epsilon_{r}=9.8$ is $43.9 \%$.

Table I reports the maximum values of normalized conductances, $G_{\text {rad }}^{\max }$, for several dielectric constants. Their actual values are important since they can be read as front to back ratios at the band extreme. For lower dielectric constants, the BW are larger, [9], however the front to back ratios are smaller, thus a preliminary trade off between the front to back ratio and the BW must be performed, even before considering the surface wave efficiency.

\section{B. Ideal $E B G$}

It is not possible to completely avoid exciting surface waves in dielectric slabs since they are intrinsically needed to satisfy the boundary conditions. However, a source will not launch real power into surface waves when it is surrounded by a cavity that guarantees the cancellation of the outgoing wave with a reflected wave of the same amplitude. In the present study, rather than cavities [10], we will consider an ideal EBG as a periodic structure operating in the bandgap to reproduce the cavity behavior.

The surface wave component of the slot admittance in presence of such ideal EBG presents real part equal to zero since no power is lost in surface waves. However at nearly all frequencies, except at resonance, the ideal EBG would induce an imaginary part to the admittance associated to surface wave reflections. The resonant point is defined by tuning the cavity dimension corresponding to the distance between the antenna and theEBG. The presence of the imaginary part of the admittance induced by the EBG significantly reduces the bandwidth with respect to the theoretically optimal case of the previous subsection.

The scope of this paper is the analysis of PCS structures as in Fig. 1 that are realized in planar technology and aim at suppressing $\mathrm{TM}_{0}$ waves only. In next section, the different components of the admittance of a slot surrounded by a planar EBG in a two-dimensional (2-D) case will be described. The deviations from the ideal behavior will be explicitly quantified.

\section{2-D MODEL}

In order to design a realistic EBG that achieves the best TM surface wave suppression it is important to establish the distance between the antenna and the EBG that best approaches the ideal EBG behavior. A simplified analytical model of the interaction between the antenna and the EBG is represented by 


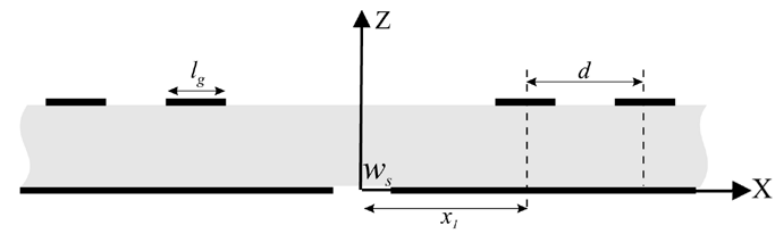

(a)

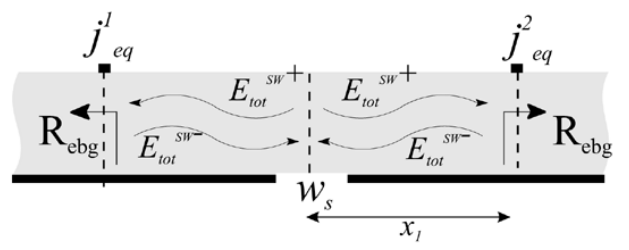

(b)

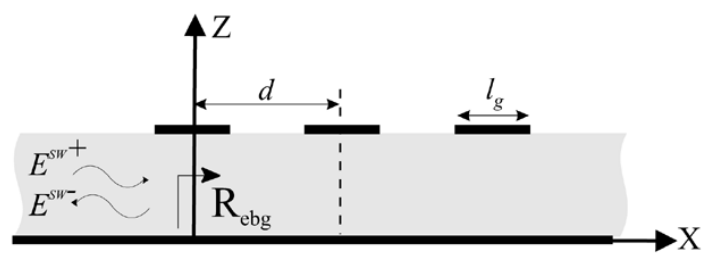

(c)

Fig. 5. 2-D geometry.

the 2-D, $y$-independent, structure of Fig. 5(a), with a TM field configuration (magnetic field along $y$ ). The 2-D structure is obtained as the cut for $\phi=0$ of the three-dimensional (3-D) structure of Fig. 1. The source is an aperture of width $w_{s}$ etched on the ground plane with current distribution $m_{s}$. The same finite number of strip gratings is printed on top of the slab at the left and the right of the aperture. The first gratings are located at a distance $x_{1}$ from the central source, which corresponds to $\rho_{1}$ of the 3-D structure (Fig. 1).

The periodicity $d$ and length of the strips $l_{g}$ are designed, as explained in [7], in order to obtain a bandgap of the $\mathrm{TM}_{0}$ mode in the frequencies from 4.8 to $6.4 \mathrm{GHz}$. The procedure is based on the solution of an integral equation derived enforcing the vanishing of the electric field (EFIE) on the metallic loadings, which are considered to be periodic with the structure infinitely extended also in $x$. The real and imaginary parts of the normalized solutions, $k / k_{0}$, of the dispersion equations pertinent to the grounded dielectric slab $\left(\epsilon_{r}=9.8, h=3.81 \mathrm{~mm}\right)$ loaded by a strip grating are shown, with continuous line, in Fig. 6. Note that the specific dielectric thickness has been chosen since it is commercially available.

Once the design of the EBG is fixed, the finite structure in Fig. 5(a), can be analyzed using a standard element by element method of moments (MoM). Given a lumped electric current along $\mathbf{i}_{x}$ as excitation of the slot, the current on all strips, the voltage on the slot, the impedance and the efficiency of the system can all be calculated as a function of the frequency. The surface wave efficiency, (1), is plotted in Fig. 7 for the slot alone, and when one, two and four strips are located on each side of the slot. Here $x_{1}$ is chosen arbitrarily to be equal to the periodicity $x_{1}=d=13.7 \mathrm{~mm}$. With the infinite model, the attenuation constant of the mode inside the bandgap can be derived, see Fig. 6, and then used as indication of the number of EBG elements necessary to attenuate the mode. A significant improvement of the efficiency is obtained in the bandgap region when

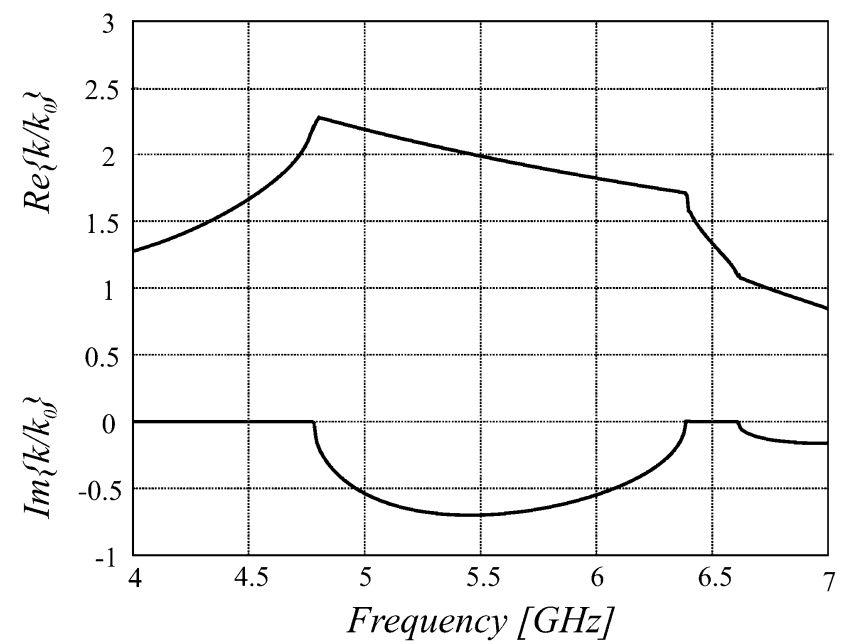

Fig. 6. Dispersion diagram for the $\mathrm{TM}_{0}$ surface wave $\left(d=13.7 \mathrm{~mm}, l_{g}=\right.$ $6.6 \mathrm{~mm}$ ).

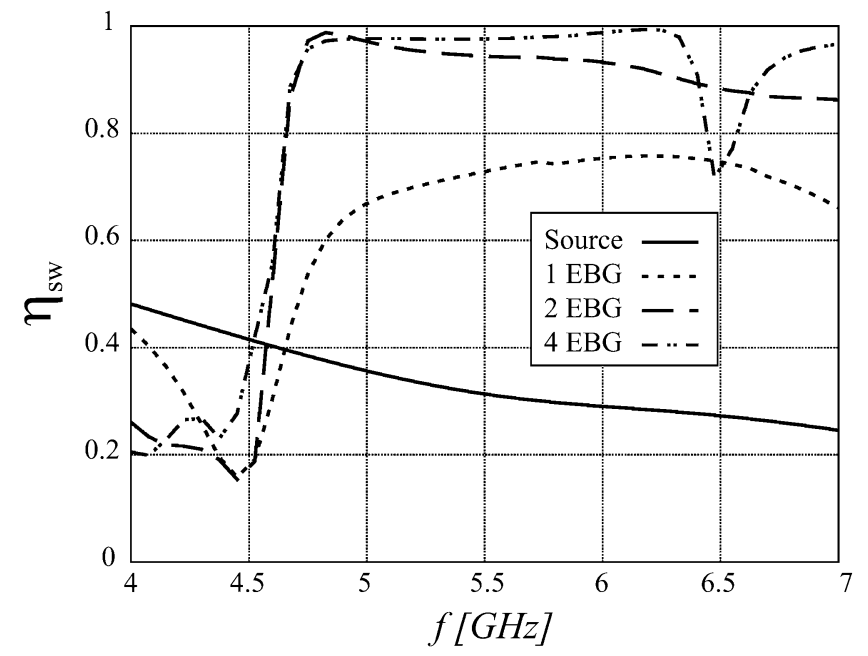

Fig. 7. Surface wave efficiency of the 2-D model without EBG and with one, two and four elements $\operatorname{EBG}\left(d=13.7 \mathrm{~mm}, l_{g}=6.6 \mathrm{~mm}, x_{1}=13.7 \mathrm{~mm}\right)$.

the number of elements goes from 1 to 2 . A smaller improvement is obtained when other two EBG elements are added. With further inclusions of elements there are no significant improvements.

\section{A. Single Mode Field Representation}

In parallel to the full wave analysis and in order to derive the physical insight necessary to determine the influence of the parameter $x_{1}$, we can set up a simplified model to characterize the wave interaction. The $x$-component of the electric field can be represented, resorting to the equivalence theorem, as the sum of the field radiated by the slot on an infinite substrate in absence of the EBG plus the field scattered by the EBG

$$
E_{\mathrm{tot}}(\mathbf{r})=E_{\mathrm{sl}}(\mathbf{r})+E_{\mathrm{ebg}}(\mathbf{r})
$$

where $\mathbf{r}=(x, z)$. In general all the spectral components are necessary to represent the $E_{\mathrm{sl}}$ in the vicinity of the antenna or $E_{\text {ebg }}$ in the vicinity of the EBG

$$
\begin{aligned}
E_{\mathrm{sl}}(\mathbf{r}) & =E_{\mathrm{sl}}^{\mathrm{sw}+}(\mathbf{r})+E_{\mathrm{sl}}^{\mathrm{rad}}(\mathbf{r}) \\
E_{\mathrm{ebg}}(\mathbf{r}) & =E_{\mathrm{ebg}}^{\mathrm{sw}+}(\mathbf{r})+E_{\mathrm{ebg}}^{\mathrm{sw}-}(\mathbf{r})+E_{\mathrm{ebg}}^{\mathrm{rad}}(\mathbf{r})
\end{aligned}
$$


where the apex rad stands for radiated field which is defined as the difference between the complete fields and their $\mathrm{TM}_{0}$ surface wave components. Note that in other contests [11], these difference fields are indicated as fringe fields rather than radiated fields. The surface wave components are indicated via the superscript + and - for outgoing or ingoing waves respectively. When the EBG field, $E_{\mathrm{ebg}}$, is observed in the vicinity of the antenna, or conversely the slot field, $E_{\mathrm{sl}}$, is observed in the vicinity of the EBG, the surface waves contributions are dominant. Thus, in the following, we will concentrate only on the surface wave contribution. The projection on the surface wave components of the total field, can be represented as a standing wave pattern [see Fig. 5(b)], for instance for the electric field along $x$

$$
E_{\mathrm{tot}}^{\mathrm{sw}}(\mathbf{r})=E_{\mathrm{sl}}^{\mathrm{sw}+}(\mathbf{r})+E_{\mathrm{ebg}}^{\mathrm{sw}+}(\mathbf{r})+E_{\mathrm{ebg}}^{\mathrm{sw}-}(\mathbf{r})
$$

The surface wave electric and magnetic fields radiated inside the slab can all be derived by capturing the residue at the surface wave pole $k_{\mathrm{sw}}$ that appears in the spectral representation of the pertinent Green's function of the substrate. All the residue expressions used in this paper are given in the Appendix. $E_{\mathrm{sl}}^{\mathrm{sw}+}$, radiated by a slot of current distribution $m_{s}$, can be expressed as

$$
E_{\mathrm{sl}}^{\mathrm{sw}+}(\mathbf{r})=j M_{s}\left(k_{\mathrm{sw}}\right) \operatorname{Res}\left(V_{\mathrm{TM}}^{m}\left(k_{\mathrm{sw}}, z\right)\right) e^{-j k_{\mathrm{sw}}|x|} .
$$

For narrow slots one can approximate the magnetic current distribution of the source, $m_{s}$, via a constant function so that $M_{s}=F T\left(m_{s}\right)$ is also known.

The surface wave electric field scattered by the EBG, $E_{\mathrm{ebg}}^{\mathrm{sw}}$, can be described, in the region $|x|<x_{1}$, resorting to two equal equivalent elementary electric currents located at $x_{1}$ and $-x_{1}$, and oriented along $x$ [see Fig. 5(b)]

$$
\mathbf{j}_{\mathrm{ebg}}^{2 / 1}=I_{\mathrm{ebg}} \delta\left(x \mp x_{1}\right) \mathbf{i}_{x}
$$

The amplitude of these currents is unknown at this stage. The surface wave electric fields radiated by these currents can be expressed, highlighting the outgoing and ingoing waves, as

$$
E_{\mathrm{ebg}}^{\mathrm{sw} \pm}(\mathbf{r})=j I_{\mathrm{ebg}} \operatorname{Res}\left(V_{\mathrm{TM}}^{j}\left(k_{\mathrm{sw}}, z\right)\right) e^{-j k_{\mathrm{sw}} x_{1}} e^{\mp j k_{\mathrm{sw}}|x|} .
$$

Using these analytical, residue based contributions, the actual value of the equivalent current amplitude $I_{\text {ebg }}$ can be obtained characterizing the EBG structure in terms of an equivalent reflection coefficient. Observing the field at $\left(x=x_{1}, z=h\right)$, one can write the reflection coefficient as the ratio between the ingoing and outgoing waves fields

$$
R_{\mathrm{ebg}}=\frac{E_{\mathrm{tot}}^{\mathrm{sw}-}}{E_{\mathrm{tot}}^{\mathrm{sw}+}}=\left.\frac{E_{\mathrm{ebg}}^{\mathrm{sw}-}(\mathbf{r})}{E_{\mathrm{sl}}^{\mathrm{sw}+}(\mathbf{r})+E_{\mathrm{ebg}}^{\mathrm{sw}+}(\mathbf{r})}\right|_{z=h} ^{x=x_{1}} .
$$

Substituting (10) and (12) into (13), one can obtain the expression for the amplitude of the equivalent current as a function of the reflection coefficient of the EBG

$$
I_{\mathrm{ebg}}=M_{s}\left(k_{\mathrm{sw}}\right) \frac{\operatorname{Res}\left(V_{\mathrm{TM}}^{m}\left(k_{\mathrm{sw}}, h\right)\right)}{\operatorname{Res}\left(V_{\mathrm{TM}}^{j}\left(k_{\mathrm{sw}}, h\right)\right)} \frac{R_{\mathrm{ebg}} e^{-j k_{\mathrm{sw}} x_{1}}}{1-R_{\mathrm{ebg}} e^{-j 2 k_{\mathrm{sw}} x_{1}}} .
$$

\section{B. 2-D-EBG Reflection Coefficient}

The important aspect of the single mode representation of the fields is that all quantities are expressed analytically once the reflection coefficient of the surface wave impinging on the EBG is known. We define the reflection coefficient with respect to the component of the electric field parallel to the direction of propagation of the wave, $\mathbf{i}_{x}$. Since the boundary condition that the EBG enforces on the electric field is $E_{i}+E_{s}=E_{\text {tot }}=0$ and assuming an ideal EBG where $E_{s}=\Gamma E_{i}^{\mathrm{sw}}$, the reflection coefficient must be $\Gamma=-1$. In real EBGs, this reflection coefficient is instead a frequency dependent complex value.

The reflection coefficient can be obtained from the analysis of the canonical problem in Fig. 5(c). Only a few elements of the EBG are retained in the analysis since the electric current distribution decreases rapidly for the elements that are further away from the source when the frequency of operation is well inside the bandgap of the structure [7]. In this canonical configuration the reflection coefficient for 2-D-EBG is defined as follows:

$$
R_{\mathrm{ebg}}=\left.\frac{E^{\mathrm{sw}}-(\mathbf{r})}{E^{\mathrm{sw}}+(\mathbf{r})}\right|_{z=h} ^{x=0}
$$

where $E^{\mathrm{sw}+}$ and $E^{\mathrm{sw}-}$ are the amplitudes of the direct and reflected $\mathrm{TM}_{0}$ surface waves, respectively.

The analysis can be performed solving the same MoM code that was used to obtain the results of Fig. 7. In this case it solves the (EFIE) that imposes the vanishing of the tangent electric field on the metallic gratings under surface wave incidence

$$
E^{\mathrm{sw}+}(\mathbf{r})=E_{0} e^{-j k_{\mathrm{sw}} x} \mathbf{i}_{x}
$$

Once the MoM has been solved, the electric current on the entire grating is known, $j_{g r}(\mathbf{r})$. One can then obtain the field $E_{\mathrm{sw}}^{-}$by first expressing the scattered field in the spectral domain and by then applying again the residue theorem to retain only the surface wave contribution at $k_{x}=-k_{\mathrm{sw}}$. The actual expression is

$$
E^{\mathrm{sw}-}(\mathbf{r})=j J_{g r}\left(-k_{\mathrm{sw}}\right) \operatorname{Res}\left(V_{\mathrm{TM}}^{j}\left(k_{\mathrm{sw}}, z\right)\right) e^{j k_{\mathrm{sw}} x}
$$

where $J_{g r}\left(k_{x}\right)$ is the Fourier transform of $j_{g r}(\mathbf{r})$.

In Fig. 8 the amplitude and phase of the reflection coefficient is shown, for the structure of Fig. 5(c) including 1, 2, or 4 elements in the $\mathrm{x}$-direction. The curve outlines the bandgap region shown in Fig. 7 via a significant change of derivative of the reflection coefficient as a function of the frequency. The reflection coefficient of real EBGs have amplitudes lower than one. However, since the surface wave efficiencies observed in Fig. 7 for the same configurations correspond to almost $100 \%$, it can be concluded that the remaining portion of the power is radiated. This radiation is responsible for an increase of the directivity of an antenna surrounded by the EBG, as was experimentally observed in [7].

\section{INPUT ADMITTANCE}

The single mode representation allows to rigorously define the surface wave admittances briefly introduced in Section II-B. Generally, these are considered more useful design parameters 

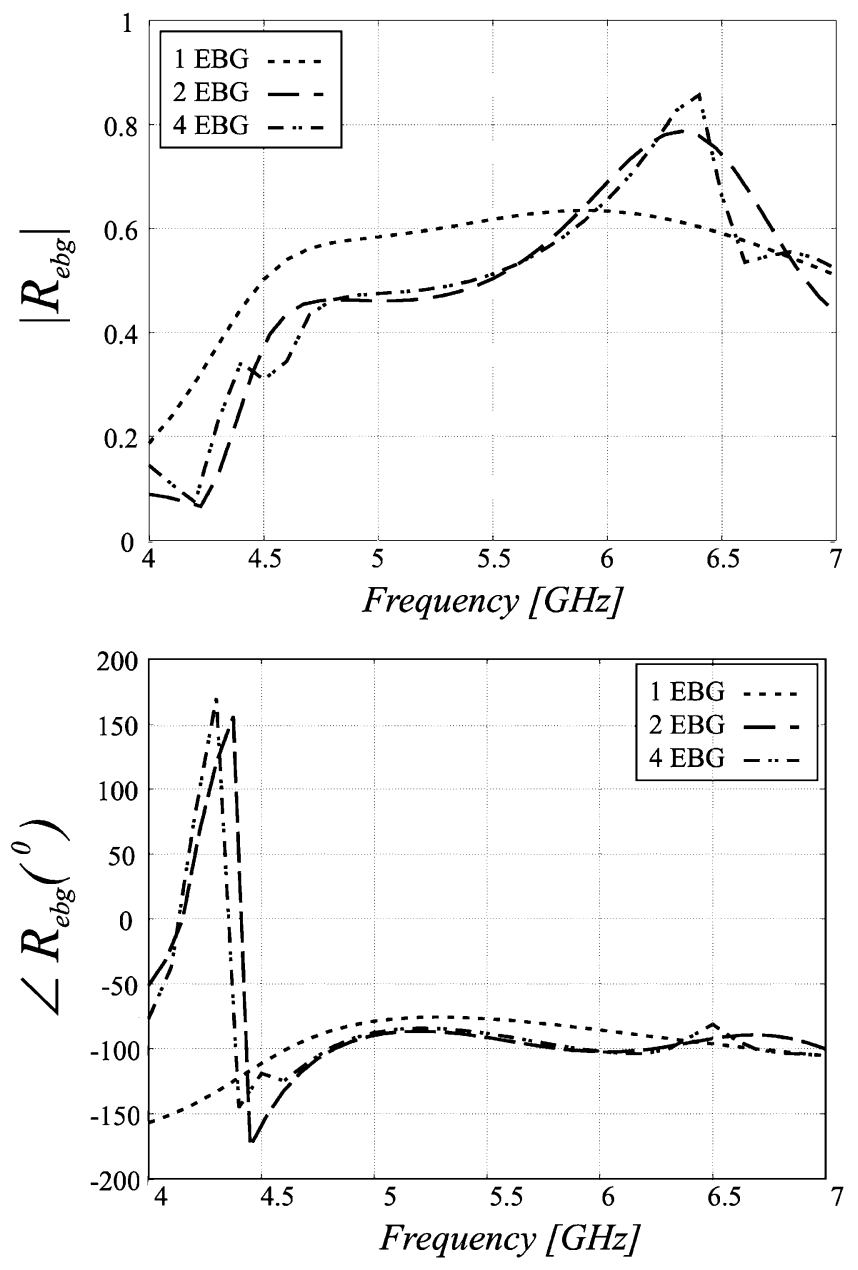

Fig. 8. Reflection coefficient for the $\mathrm{TM}_{0}$ surface wave $\left(d=13.7 \mathrm{~mm}, l_{g}=\right.$ $6.6 \mathrm{~mm}$ ) for different number of EBG elements.

than the field themselves. The admittance can be expressed in the space domain as

$$
Y=-\int_{-w_{s} / 2}^{w_{s} / 2} m_{s}(x) H(x) d x
$$

where $m_{s}$ is the magnetic current distribution on the slot and $H$ is the tangent magnetic field (along $y$ ). Then, the total input admittance of the slot can be expressed as the superposition of two reaction integrals. The first involves the magnetic field in absence of the EBG for an infinite substrate, while the second one involves the field radiated by the equivalent EBG currents. Accordingly, an appropriate representation is

$$
Y_{\mathrm{tot}}=Y_{\mathrm{sl}}+Y_{\mathrm{ebg}}
$$

This expression is the circuit equivalent of (6). In the self reaction $Y_{\mathrm{sl}}$ it is possible to distinguish a surface wave term and a radiated term

$$
Y_{\mathrm{sl}}=Y_{\mathrm{sl}}^{\mathrm{sw}+}+Y_{\mathrm{sl}}^{\mathrm{rad}}
$$

The term $Y_{\mathrm{sl}}^{\mathrm{sw}+}$ can be calculated analytically resorting to standard slab's GF theory and applying the residues theorem

$$
Y_{\mathrm{sl}}^{\mathrm{sw}+}=G_{\mathrm{sl}}^{\mathrm{sw}+}=-j M_{s}^{2}\left(k_{\mathrm{sw}}\right) \operatorname{Res}\left(I_{\mathrm{TM}}^{m}\left(k_{\mathrm{sw}}, 0\right)\right) \text {. }
$$

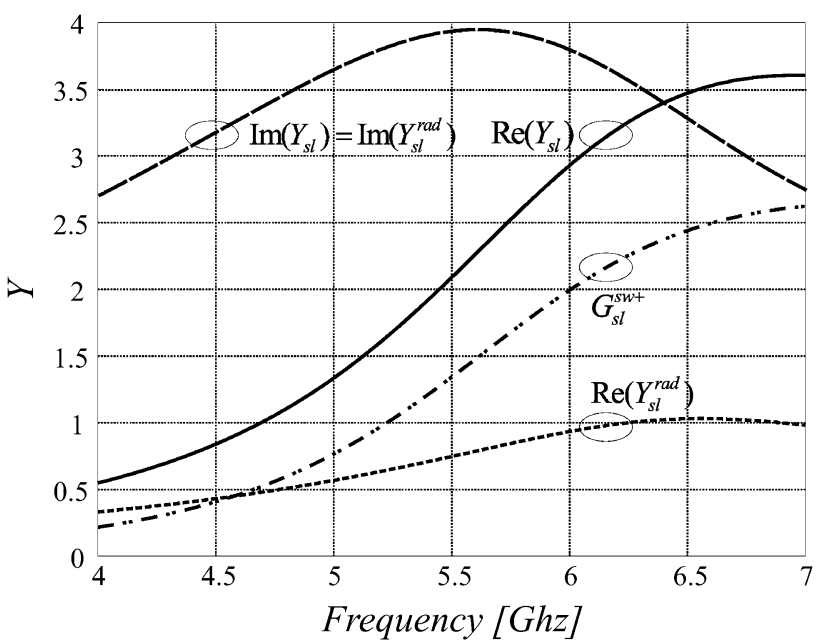

Fig. 9. Real and imaginary part of the input admittance of a slot with $w_{s}=1.5$ $\mathrm{mm}$ in absence of the EBG.

Notice that the surface wave contribution is completely real since all the power launched by the slot in surface waves is lost in the substrate which is assumed infinitely extended.

In Fig. 9 the real and imaginary part of the total slot admittance in absence of an EBG is shown together with its two components: the surface wave $G_{\mathrm{sl}}^{\mathrm{sw}+}$ and the radiated $Y_{\mathrm{sl}}^{\mathrm{rad}}$ one. One can note that $G_{\mathrm{sl}}^{\mathrm{sw}+}>Y_{\mathrm{sl}}^{\mathrm{rad}}$ is an explicit manifestation of the low efficiency of the slot alone.

The reaction, $Y_{\mathrm{ebg}}$, can be expressed via a surface wave contribution only, for $x_{1}$ sufficiently large, i.e., $Y_{\mathrm{ebg}} \approx Y_{\mathrm{ebg}}^{\mathrm{sw}}$

$$
Y_{\mathrm{ebg}}^{\mathrm{sw}}=-j 2 I_{\mathrm{ebg}} M_{s}\left(k_{\mathrm{sw}}\right) \operatorname{Res}\left(I_{\mathrm{TM}}^{j}\left(k_{\mathrm{sw}}, 0\right)\right) e^{-j k_{\mathrm{sw}} x_{1}}
$$

where the factor 2 is due to the presence of the two equivalent EBG currents.

Substituting in (22) the expressions of the residues (see Appendix) and the equivalent current amplitude (14), then using (21) one can derive the following relation:

$$
Y_{\mathrm{ebg}}^{\mathrm{sw}}=2 G_{\mathrm{sl}}^{\mathrm{sw}+} \frac{R_{\mathrm{ebg}}}{e^{j 2 k_{\mathrm{sw}} x_{1}}-R_{\mathrm{ebg}}} .
$$

This analytical relation explicitly shows how the impact of $x_{1}$ on the admittance of the slot is modulated by $R_{\mathrm{ebg}}$.

Now we can clarify the ideal EBG concept that was mentioned in Section II-B. In fact for $x_{1}=\lambda_{\text {sw }} / 2$ and assuming an EBG that realizes a short circuit for the impinging surface wave, $R_{\text {ebg }}=-1$, the EBG contribution to the surface wave admittance, (23), becomes

$$
Y_{\mathrm{ebg}-\text { ideal }}^{\mathrm{sw}}=-G_{\mathrm{sl}}^{\mathrm{sw}+} .
$$

This expression describes the situation in which all the impinging surface wave power is reflected from the EBG toward the slot with such a phase to cancel the outgoing surface wave. The real part of $Y_{\mathrm{ebg}-\text { ideal }}^{\mathrm{sw}}$ is equal to $-G_{\mathrm{sl}}^{\mathrm{sw}+}$ for all frequencies, while its imaginary part is equal to zero at least for one frequency point. In that frequency point, only $Y_{\mathrm{sl}}^{\mathrm{rad}}$, which is essentially associated to the radiated space wave, would remain to be accounted for. Fig. 10 shows the admittances of the slot for the cases of ideal EBG and real EBG using the same value of $x_{1}=\lambda_{\mathrm{sw}} / 2 \approx 21 \mathrm{~mm}$. In the real EBG case, the real part 


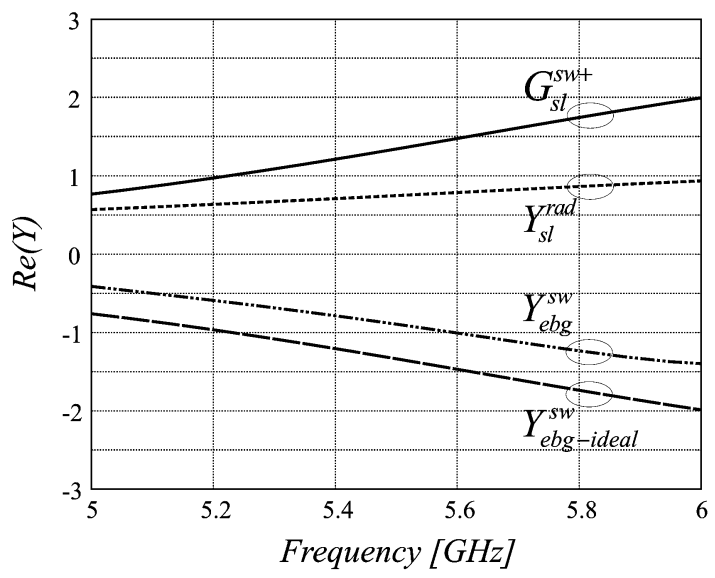

(a)

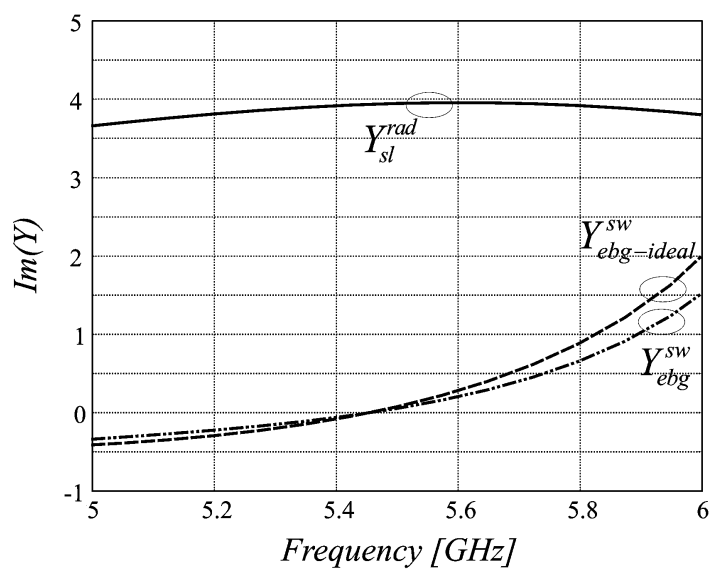

(b)

Fig. 10. Real and imaginary parts of the input admittance of the slot for an ideal EBG and real EBG $\left(x_{1}=21 \mathrm{~mm}\right)$.

of surface wave admittance associated to an infinitely extended slab is not completely cancelled by the reflected waves.

Fig. 11 plots the EBG surface wave admittance, (22), the slot admittance, (20), and sum of the two, $Y_{\text {tot }}$ as a function of $x_{1}$. The frequency selected is $f=5.5 \mathrm{GHz}$ at the center of the band. The total wave admittance when the EBG is present oscillates around the value of the admittance in absence of the EBG. It can be observed that the minimum value of the real part of the total admittance is obtained when $x_{1}=\lambda_{\mathrm{sw}} / 2=20.7 \mathrm{~mm}$ (i.e., half of the surface wave length).

Using (19), in which $Y_{\mathrm{sl}}$ is evaluated full wave and $Y_{\mathrm{ebg}}$ with the analytical approximation in (23), one can evaluate the input impedance of the antenna in the presence of the EBG, Fig. 12. To validate the formulation, the same structure has also been analyzed with the standard 2-D el. by el. MoM. The comparison is very good highlighting that the impact of the EBG on the input impedance is very well described by the surface wave interactions only. In the same figure, also the impedance considering an ideal EBG is plotted. In this specific case, even if the actual value of the total impedance is derived using the ideal reflection coefficient, the resonant frequency point is nevertheless well approximated.

\section{ApPlicability of the Single Mode RePRESEntation}

In the previous section, a 2-D model has been developed to analyze the effect of the EBG on the admittance of a slot. More-

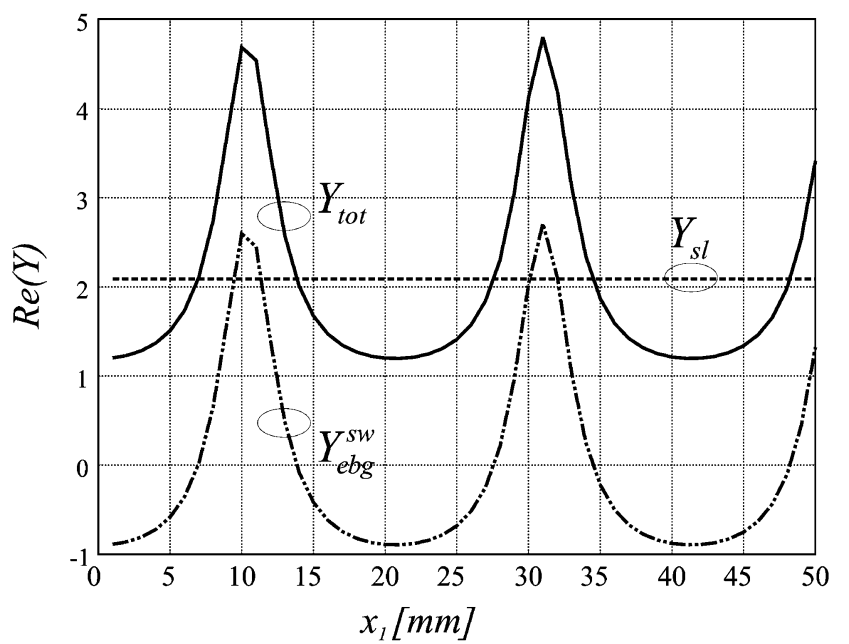

(a)

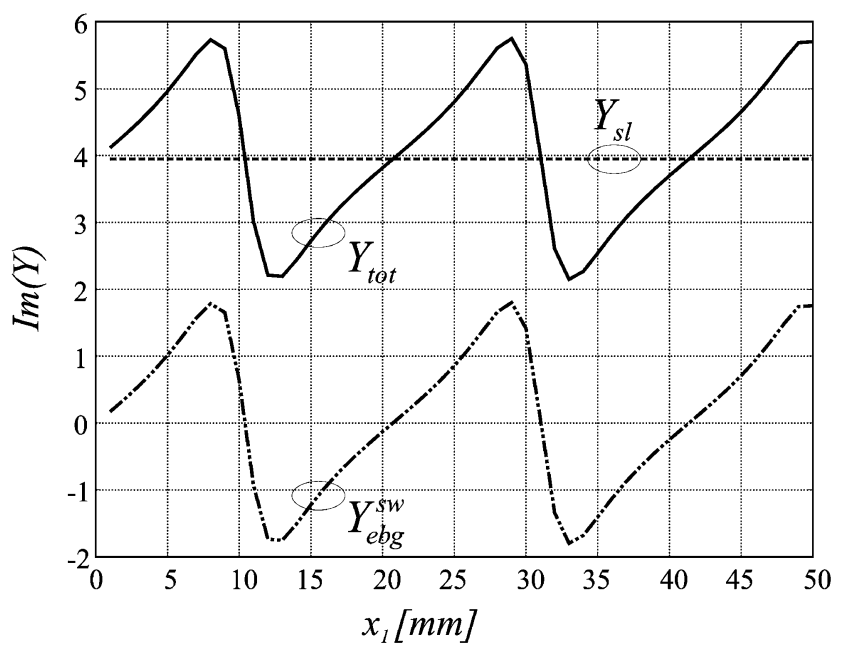

(b)

Fig. 11. Real and imaginary parts of the input admittance as a function of $x_{1}$ at $f=5.5 \mathrm{GHz}$.

over the model was based on a single mode interaction that is assumed valid for large values of $x_{1}$ in the 2-D case. Both these aspects would seem to limit the applicability of the results presented in realistic (3-D) configurations where the EBG is so close to the antenna that the $\mathrm{TM}_{0}$ surface wave is not sufficient to describe the complete interaction.

However the important aspect of the previous field representation is not the quantitative one, there are full wave simulators for that. The key issue is that the physical insight of the single mode interaction guides the initial slot-EBG design already very close to the final one. Even without knowing the actual value of the reflection coefficient, but just thinking in terms of an ideal EBG, one knows that the radius $\rho_{1}$, in configurations as those in Fig. 1, has to be close to half of the surface wave wavelength for maximum radiation bandwidth. The actual phase of the reflection coefficient will only moderately affect the value of $\rho_{1}$. Moreover, the amplitude of the reflection coefficient being lower than unity does not really play a major role besides giving some indication on how much radiation emerges from the EBG.

To emphasize this design aspect, three different slots in the presence of PCS-EBG configurations (see Fig. 1) have been investigated using Ansoft Designer. The slabs are the same in all the cases. The radii were $\rho_{1}=10 \mathrm{~mm}, \rho_{1}=18 \mathrm{~mm}$ 


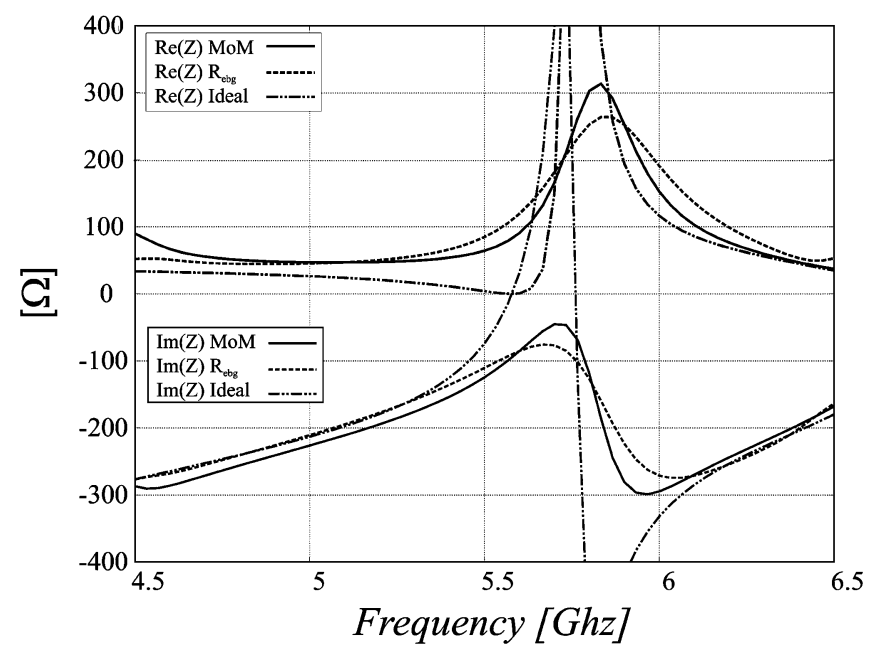

Fig. 12. Real and imaginary part of the input impedance of a slot in presence of 2 elements EBG calculated with 2-D el. by el. MoM, using the reflection coefficient at the real EBG and at the ideal EBG $\left(d=13.7 \mathrm{~mm}, l_{g}=6.6 \mathrm{~mm}\right.$, $x_{1}=30 \mathrm{~mm}$ ).

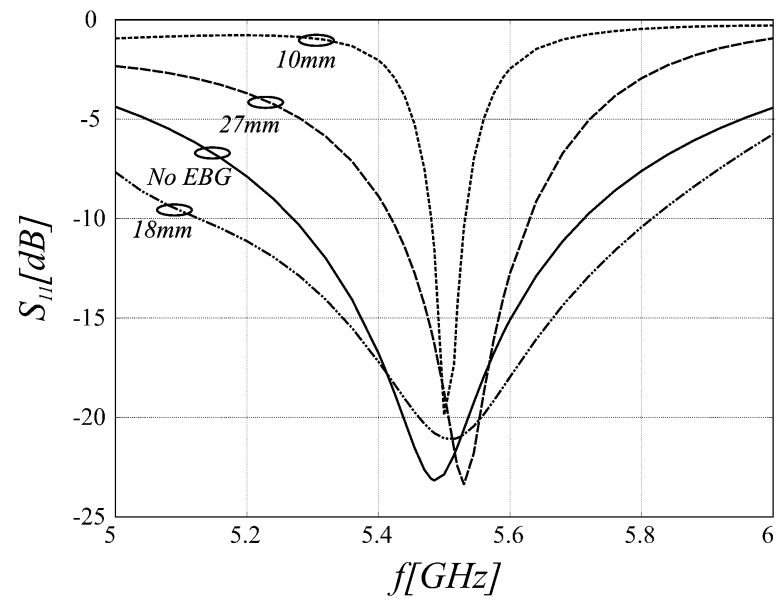

Fig. 13. Reflection coefficients for $\rho_{1}=10 \mathrm{~mm}, \rho_{1}=18 \mathrm{~mm}$ and $\rho_{1}=27$ $\mathrm{mm}$.

and $\rho_{1}=27 \mathrm{~mm}$, roughly corresponding to $\lambda_{\mathrm{sw}} / 4, \lambda_{\mathrm{sw}} / 2$ and $3 / 4 \lambda_{\mathrm{sw}} \approx \lambda_{0} / 2$. In order to perform a fair comparison between the different configurations the central slots were tuned in the three cases to achieve the best match at the central frequency of $5.5 \mathrm{GHz}$ when fed by a $50 \Omega$ microstrip line extended beyond the slot in an open circuit stub, see Fig. 2. The reflection coefficients pertinent to these three configurations are shown in Fig. 13 together with the reflection of the antenna without EBG. It is apparent that the largest bandwidth, around $13 \%$ at -10 $\mathrm{dB}$, is obtained with $\rho_{1} \approx \lambda_{\mathrm{sw}} / 2$. In this case the $\mathrm{BW}$ is even larger than the one of the slot in absence of the EBG (a case with surface wave efficiency less than $40 \%$, see Fig. 7). Much lower BWs are obtained for the other radii. In the case of $\rho_{1}=\lambda_{0} / 2$ case, the BW is reduced because the reflections from the EBG return in phase with the outgoing surface waves. For the smallest radius case, the BW is even smaller as the reactive coupling between the slot and the EBG has also a significant effect on the frequency behavior.

On may note that $18 \mathrm{~mm}$ is not exactly half of the surface wave wavelength at the center of the bandwidth. It was the value selected for the antenna prototype in [7]. In a realistic case, the

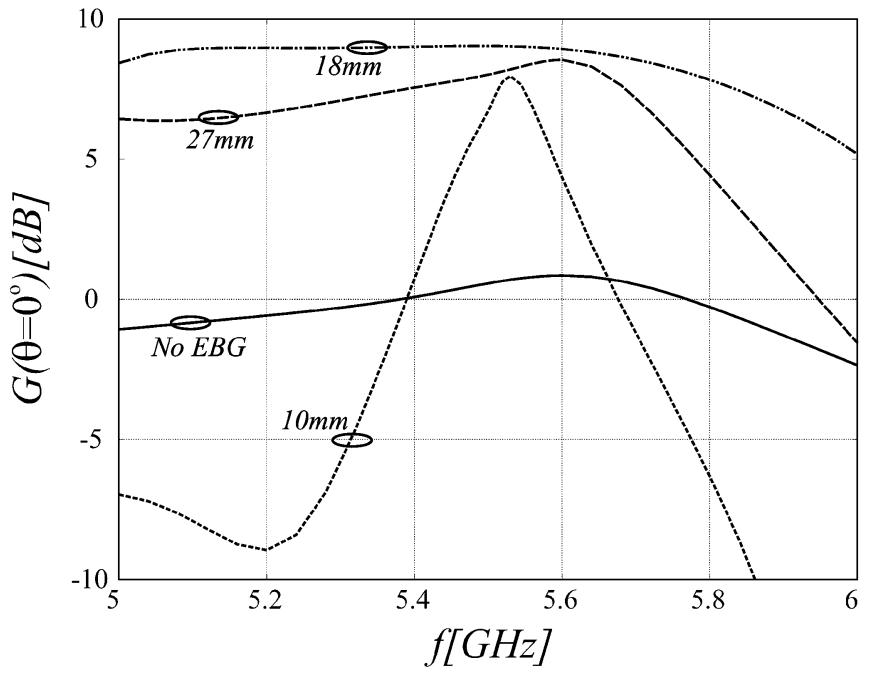

Fig. 14. Gain for $\rho_{1}=10 \mathrm{~mm}, \rho_{1}=18 \mathrm{~mm}$ and $\rho_{1}=27 \mathrm{~mm}$.

difference between $18 \mathrm{~mm}$ and $20.7 \mathrm{~mm}$ is the range of approximation of the simplified design rule, $\rho_{1}=\lambda_{\text {sw }} / 2$.

The gain calculated by Ansoft Designer can be interpreted as a measure of the radiation efficiency of the antenna. Fig. 14 shows the gains for the four configurations at broadside as a function of the frequency. The antenna without EBG has a very low gain due to the fact that more than $60 \%$ of the power is lost into the $\mathrm{TM}_{0}$ surface wave (Ansoft Designer considers an infinitely extended substrate). The case of $\rho_{1} \approx \lambda_{\text {sw }} / 2$ provides the highest gain overall the frequency band. The case of $\rho_{1}=$ $\lambda_{0} / 2$ provides essentially the same gain of $\rho_{1} \approx \lambda_{\mathrm{sw}} / 2$ at the central frequency, but lower gain in average over the complete band. The fact that a distance between the antenna and the EBG of $\rho_{1} \approx \lambda_{0} / 2$ results in a higher gain was emphasized also in [12] for a single soft-surface structure.

\section{CONCLUSION}

The role of the distance between a central resonant antenna and a surrounding EBG structure printed on the same slab has been addressed in this paper. The optimal radiation bandwidth for a printed slot surrounded by an PCS-EBG is achieved when the inner radius, $\rho_{1}$, of the equivalent EBG cavity is approximately half of the first surface wave wavelength $\lambda_{\mathrm{sw}} / 2$. In this situation the wave reflected from the EBG cancels out the outgoing waves emanated by the source. At least in first approximation all the power delivered to the slot is then radiated. Any other configuration reduces the $\mathrm{BW}$. If $\rho_{1}$ is too small the coupling between the slot and the EBG is reactive and the field around the slot does not have sufficient room to develop itself into radiation. If $\rho_{1}$ is too large the SWs reflected from the EBG add in phase with the ones launched by the slot. Thus the optimal value of $\rho_{1}$ can be tuned using a single mode model that characterizes the interaction of the SW with a planar EBG by means of a reflection coefficient. The procedure was demonstrated to be accurate and close to analytical in a simple 2-D case. It is possible to extend the approach to more realistic 3-D structures. However, for simple planar antenna+EBG structures full wave commercial tools are fast and accurate enough to be used in the 


$$
\begin{aligned}
D_{\mathrm{TM}}^{\prime}\left(k_{\mathrm{SW}}\right) & =k_{\mathrm{sw}} \times \frac{k_{z 0} \sin \left(k_{z} h\right)\left(k_{z 0} \epsilon_{r} h-j\right)-k_{z} \cos \left(k_{z} h\right)\left(\epsilon_{r}-j k_{z 0} h\right)}{k_{z 0} k_{z}} \\
k_{z} & =\sqrt{k_{0}^{2} \epsilon_{r}-k_{\mathrm{sw}}^{2}} \\
k_{z 0} & =-j \sqrt{k_{\mathrm{sw}}^{2}-k_{0}^{2}}
\end{aligned}
$$

final refinement phase, if the initial design, based on the 2-D analysis, is already close to the final one.

\section{APPENDIX}

The surface wave electric and magnetic fields radiated inside the slab can be derived by applying the residues theorem at the surface wave pole $k_{\mathrm{sw}}$ to the spectral representation of the field using the Green's function of the substrate. If a function $F\left(k_{\rho}\right)=\left(N\left(k_{\rho}\right)\right) /\left(D\left(k_{\rho}\right)\right)$ has a pole in $k_{\rho}=k_{\mathrm{sw}}$, the residues can be calculated as $\operatorname{Res}\left(F\left(k_{\rho}\right)\right)=\left(F\left(k_{\mathrm{sw}}\right)\right) /\left(\left.D^{\prime}\left(k_{\rho}\right)\right|_{k_{\rho}=k_{\mathrm{sw}}}\right)$.

The residue of the voltage solution inside the slab, $z \leq h$, of the TM transmission line associated to the grounded slab with a series voltage source at the ground plane, used in (10), has the following expression:

$$
\operatorname{Res}\left(V_{\mathrm{TM}}^{m}\left(k_{\mathrm{sw}}, z\right)\right)=\frac{\sin \left(k_{z} z\right)}{\sin \left(k_{z} h\right)} \frac{\epsilon_{r} k_{z 0}}{D_{\mathrm{TM}}^{\prime}\left(k_{\mathrm{sw}}\right)}
$$

where, see the equation at the top of the page.

The residue of the voltage solution at $z \leq h$ of the TM transmission line associated to the grounded slab with a parallel current source at $z=h$, used in (12) and (17), has the following expression:

$$
\operatorname{Res}\left(V_{\mathrm{TM}}^{j}\left(k_{\mathrm{sw}}, z\right)\right)=j \frac{\zeta_{0} k_{z 0} k_{z}}{k_{0}} \frac{\sin \left(k_{z} z\right)}{D_{\mathrm{TM}}^{\prime}\left(k_{\mathrm{sw}}\right)}
$$

where $D_{\mathrm{TM}}^{\prime}\left(k_{\mathrm{sw}}\right), k_{z}$ and $k_{z 0}$ are the same as in (25).

The residue of the current solution at $z=0$ of the TM transmission line associated to the grounded slab with a series voltage source at the ground plane, appearing in (21), assumes the form

$$
\operatorname{Res}\left(I_{\mathrm{TM}}^{m}\left(k_{\mathrm{sw}}, 0\right)\right)=j \frac{\epsilon_{r} k_{0}}{\zeta_{0} k_{z} \sin \left(k_{z} h\right)} \frac{\epsilon_{r} k_{z 0}}{D_{\mathrm{TM}}^{\prime}\left(k_{\mathrm{SW}}\right)}
$$

where $D_{\mathrm{TM}}^{\prime}\left(k_{\mathrm{sw}}\right), k_{z}$ and $k_{z 0}$ are the same as in (25).

The residue of the current solution at $z=0$ of the TM transmission line associated to the grounded slab with a parallel current source at $z=h$, appearing in (22), can be expressed as

$$
\operatorname{Res}\left(I_{\mathrm{TM}}^{j}\left(k_{\mathrm{sw}}, 0\right)\right)=-\frac{\epsilon_{r} k_{z 0}}{D_{\mathrm{TM}}^{\prime}\left(k_{\mathrm{sW}}\right)}
$$

where $D_{\mathrm{TM}}^{\prime}\left(k_{\mathrm{Sw}}\right), k_{z}$, and $k_{z 0}$ are the same as in (25).

\section{ACKNOWLEDGMENT}

Welcome to the world Giuliano (Totonno) Neto.

\section{REFERENCES}

[1] P. de Maagt, R. Gonzalo, Y. C. Vardaxoglou, and J. M. Baracco, "Electromagnetic bandgap antennas and components for microwave and (sub)millimeter wave applications," IEEE Trans. Antennas Propag., vol. 51, no. 10, pp. 2667-2677, Oct. 2003.

[2] P. Baccarelli, P. Burghignoli, G. Lovat, and S. Paulotto, "A novel printed leaky-wave 'bull-eye' antenna with suppressed surface-wave excitation," in Proc. IEEE Antennas and Propagation Soc. Symp., vol. 1, Jun. 20-25, 2004, pp. 1078-1081.

[3] F. Yang and Y. Rahmat-Samii, "Microstrip antennas integrated with electromagnetic band-gap (EBG) structures: A low mutual coupling design for array applications," IEEE Trans. Antennas Propag., vol. 51, no. 10, pp. 2936-2946, Oct. 2003.

[4] Y. Fu and N. Yuan, "Elimination of scan blindness in phased array of microstrip patches using electromagnetic bandgap materials," Antennas Wireless Propag. Lett., vol. 3, pp. 63-65, 2004.

[5] R. Coccioli, Y. Fei-Ran, M. Kuang-Ping, and T. Itoh, "Aperture-coupled patch antenna on UC-PBG substrate," IEEE Trans. Microw. Theory Tech., vol. 47, no. 11, pp. 2123-2130, Nov. 1999.

[6] Z. Iluz, R. Shavit, and R. Bauer, "Microstrip antenna phased array with electromagnetic bandgap substrate," IEEE Trans. Antennas Propag., vol. 52, no. 6, pp. 1446-1453, Jun. 2004.

[7] N. Llombart, A. Neto, G. Gerini, and P. J. de Maagt, "Planar circularly symmetric EBG structures for reducing surface waves in printed antennas," IEEE Trans. Antennas Propag., vol. 53, no. 10, pp. 3210-3218, Oct. 2005.

[8] N. G. Alexopoulus, P. B. Katehi, and D. B. Rutledge, "Substrate optimization for integrated circuit antennas," IEEE Trans. Microw. Theory Tech., vol. 31, no. 7, pp. 550-557, Jul. 1983.

[9] C. A. Balanis, Antenna Theory Analysis and Design, New York: Wiley, 1997, ISBN 0-471-59 268-4.

[10] S. A. Long, "A mathematical model for the impedance of the cavitybacked slot antenna," IEEE Trans. Antennas Propag., vol. AP-25, no. 6, pp. 829-833, Nov. 1977.

[11] S. Bruni, N. Llombart, A. Neto, and G. Gerini, "Problem-matched basis functions for microstrip coupled slot arrays based on transmission line green's functions (TLGF)," IEEE Trans. Antennas Propag., vol. 53, no. 11, pp. 3556-3567, Nov. 2005.

[12] O. Li, G. DeJean, M. M. Tentzeris, J. Papapolymerou, and J. Laskar, "Radiation pattern improvement of patch antennas on a large size substrate using a compact soft-surface structure and its realization on LTCC multilayer technology," IEEE Trans. Antennas Propag., vol. 53, no. 1, pp. 200-208, Jan. 2005.

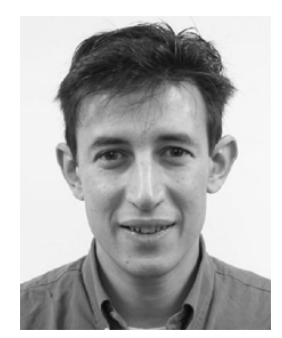

Andrea Neto (M'00) received the Laurea degree (summa cum laude) in electronic engineering from the University of Florence, Florence, Italy, in 1994 and the Ph.D. degree in electromagnetics from the University of Siena, Siena, Italy, in 2000.

Part of his Ph.D. was developed at the European Space Agency Research and Technology Center, Noordwijk, The Netherlands, where he worked for over two years in the Antenna Section. From 2000 to 2001, he was a Postdoctoral Researcher at the California Institute of Technology, Pasadena, working for the S.W.A.T. Group of the Jet Propulsion Laboratory, Pasadena. Since 2002, he has been a Senior Antenna Scientist at the Defence, Security and Safety Institute of the Netherlands Organization for Applied Scientific Research (TNO), The Hague, The Netherlands. His research interests are in the analysis and design of antennas, with emphasis on arrays, dielectric lens antennas, wideband antennas and EBG structures. 


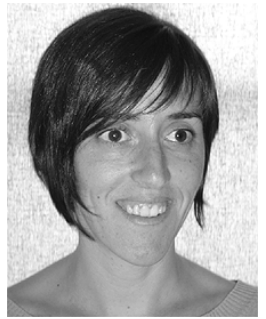

Nuria Llombart (M'06) received the Ingeniero de Telecomunicación degree from the Universidad Politécnica de Valencia, Spain, in 2002. She is working toward the Ph.D. degree at the same university.

She spent one year, 2000 to 2001, at the FriedrichAlexander University of Erlangen-Nuremberg, Germany, and worked at the Fraunhofer Institute for Integrated Circuits in Erlangen, Germany, from 2000 until 2002. Her current research interests include numerical and analytical methods for the analysis and design of printed antennas and EBG structures.

Ms. Llombart Ph.D. studies are financed and hosted by the Defence, Security and Safety Institute of the Netherlands Organization for Applied Scientific Research (TNO), The Hague, The Netherlands.

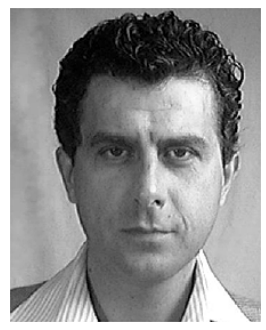

Giampiero Gerini (M'92) received the M.S. degree (summa cum laude) and the Ph.D. degree in electronic engineering from the University of Ancona, Ancona, Italy, in 1988 and 1992, respectively.

From 1994 to 1997, he was Research Fellow at the European Space Research and Technology Centre (ESA-ESTEC), Nooordwijk, The Netherlands, where he joined the Radio Frequency System Division. Since 1997, he has been with the Defence, Security and Safety Institute of the Netherlands Organization for Applied Scientific Research (TNO), The Hague, The Netherlands, where he is currently Chief Senior Scientist of the Antenna Unit in the Transceivers and Real-time Signal Processing Department. His main research interests are phased array antennas, frequency selective surfaces, and integrated front-ends.

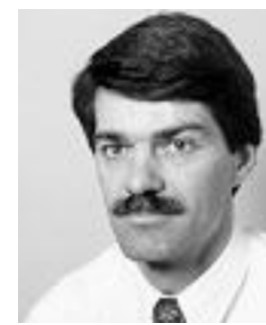

Peter de Maagt (S'88-M'88-SM'02) was born in Pauluspolder, The Netherlands, in 1964. He received the M.Sc. and Ph.D. degrees from Eindhoven University of Technology, Eindhoven, The Netherlands, in 1988 and 1992, respectively, both in electrical engineering.

$\mathrm{He}$ is currently with the European Space Research and Technology Centre (ESTEC), European Space Agency, Noordwijk, The Netherlands. His research interests are in the area of millimeter and submillimeter-wave reflector and planar integrated antennas, quasioptics, photonic bandgap antennas, and millimeter- and sub-millimeter-wave components.

He was co-recipient of the H.A. Wheeler award of the IEEE Antennas and Propagation Society for the best applications paper of the year 2001. He was granted a European Space Agency award for innovation in 2002. 